\title{
Increased platelet reactivity and significant changes in coagulation markers after cavopulmonary connection
}

H B Ravn, V E Hjortdal, E V Stenbog, K Emmertsen, O Kromann, J Pedersen, K E Sorensen

\begin{abstract}
Objective-To evaluate platelet reactivity and coagulation markers after surgical palliation of univentricular hearts.

Design and patients-Cross sectional survey of 24 patients, median age 11 (range 4-22) years, at 2 (range 0.5-6) years after a total cavopulmonary connection (TCPC; $\mathrm{n}=14$ ) or a bidirectional Glenn anastomosis (Glenn; $\mathrm{n}=10$ ).

Main outcome measures-Platelet reactivity and/or coagulation markers were measured in 20 patients (four excluded because of anticoagulant treatment) and compared with 33 healthy controls, median age 12 (range $6-16$ ) years.

Results-None of the patients had clinically apparent thromboembolic events. However, increased platelet reactivity was observed ex vivo both after collagen induced platelet aggregation (median $73 \%$ (interquartile range $61-84 \%$ ) in patients, and $61 \%(47-69 \%$ ) in controls; $\mathrm{p}<0.01)$, and after ADP induced platelet aggregation $(69 \%(53-77 \%)$ in patients, and $56 \%$ $(40-66 \%)$ in controls; $p<0.05)$. Concentrations of protein $S$ antigen, antithrombin III, and protein $\mathrm{C}$ activity were reduced after both TCPC and Glenn. A concomitant decrease was seen in coagulation factor II, VII, X, and factor VII clot activity.

Conclusions-Several abnormalities in the coagulation system were observed after bidirectional Glenn anastomosis, similar to alterations previously described in Fontan operated and TCPC patients. Antithrombotic treatment in these patients is still an unresolved issue, but aspirin is often recommended. This study shows that such a strategy is rational and the results suggest that antiplatelet treatment may be advantageous, either alone or in combination with oral anticoagulant treatment.

(Heart 2001;85:61-65)
\end{abstract}

Keywords: Fontan procedure; thrombosis; platelets; coagulation

The Fontan operation was introduced in 1971 as a surgical repair for tricuspid atresia. ${ }^{1}$ Subsequently, the Fontan principle has been widely adopted as final palliation for a variety of complex congenital heart defects. Although this procedure has increased survival and improved quality of life for these patients, various long term problems associated with the Fontan circulation have become increasingly apparent.

As the Fontan circulation operates at high systemic venous pressure, hepatomegaly with ascites, ${ }^{23}$ liver fibrosis, ${ }^{4}$ and protein losing enteropathy ${ }^{5}$ have not surprisingly been reported. Thromboembolic events have been another serious complication, occurring weeks to years postoperatively. The incidence of thromboembolism has been reported to range from $3-20 \%{ }^{7-12}$ and thrombosis occurs on both the venous and the arterial side of the circulation, although arterial thromboembolic events are thought to be caused by systemic embolisation through right to left shunts. ${ }^{13}$

Several mechanisms are likely to be responsible for the increased propensity to thrombosis after the Fontan operation. Various abnormalities in the coagulation system have been identified, including deficiency of the naturally occurring anticoagulants protein $\mathrm{C}$, protein $\mathrm{S}$, and antithrombin III. ${ }^{14-16}$ This observation has led to the recommendation that oral anticoagu- lant treatment should be given, either generally or in selected subgroups. In view of the serious haemorrhagic complications associated with oral anticoagulants, antiplatelet drugs such as aspirin have been suggested as an alternative,,$^{7817}$ although the rationale for such a strategy is not clear.

A bidirectional Glenn anastomosis or hemiFontan procedure ${ }^{18}$ can under certain circumstances be an alternative to total cavopulmonary connection (TCPC). ${ }^{19}$ Patients will achieve excellent palliation at a lower surgical risk and perhaps also avoid some of the long term sequelae mentioned above. It is unknown whether the coagulation abnormalities observed after Fontan or TCPC are associated with liver dysfunction or occur independently of this. Evaluation of the coagulation system in Glenn patients may help elucidate this issue.

Our purpose in the present study was therefore twofold - first, to evaluate platelet function in patients with a cavopulmonary connection, as this part of the haemostatic system has never been explored; and second, to investigate coagulation abnormalities following both TCPC and Glenn anastomosis. These aims were pursued by comparing platelet reactivity and coagulation markers in TCPC and Glenn patients with those observed in healthy children. 
Methods

PATIENT CHARACTERISTICS

Between March 1995 and October 1997, patients who had previously undergone a cavopulmonary operation and attended the outpatient clinic were offered cardiac catheterisation to assess postoperative status, and if willing were asked to consent to an examination of their coagulation status and platelet function. Of 24 patients seen, 20 (83\%) consented to the study. All had undergone a cavopulmonary operation around two years earlier (range 0.5-6). Ten were female and 14 male, with a median age of 11 years (range 4-22 years). Eleven patients had tricuspid atresia, eight had double inlet left ventricle, and five had other forms of complex univentricular heart malformations. Fourteen patients (58\%) had had a TCPC and $10(42 \%)$ a Glenn anastomosis. Previous palliations included systemic-pulmonary shunts in eight, pulmonary artery banding in four, and atrial septectomy in two. The remaining 10 patients had not required previous palliative surgery. All were in New York Heart Association (NYHA) class 1-2, with unobstructed pathways and no angiographic evidence of collateral formation.

Four patients (two TCPC and two Glenn) declined cardiac catheterisation, but agreed to be included in the platelet study. Of the 20 patients, four (all TCPC) were excluded because they were already on oral anticoagulant treatment and two others (Glenn) for logistic reasons. Informed consent was obtained from each subject or parent, and the study was approved by the regional ethics committee.

BLOOD SAMPLING AND HAEMODYNAMIC

VARIABLES

Blood samples for coagulation markers were obtained during cardiac catheterisation. Citrated blood for measurement of platelet aggregation and serum thromboxane $\mathrm{B}_{2}$ was obtained by clean venepuncture in all patients except four, who had blood withdrawn from the femoral sheath during cardiac catheterisation. Platelet function did not appear to be influenced by blood withdrawal from a sheath, as aggregation results were evenly distributed with both sampling techniques (data not shown). Control blood samples were obtained by clean venepuncture from 33 healthy children, 16 female and 17 male, mean age 12 years (range $6-16$ years).

Haemodynamics were evaluated by mean pressure in the pulmonary artery, superior vena cava, inferior vena cava (only in TCPC patients), and by aortic saturation (all measured during cardiac catheterisation).

\section{PLATELET AGGREGATION AND SERUM}

THROMBOXANE

Platelet rich plasma (PRP) was obtained from citrated blood samples by centrifugation at $1000 \mathrm{rpm}$ for 10 minutes at room temperature. Following removal of the supernatant plateletpoor plasma (PPP) was prepared by centrifugation at $3000 \mathrm{rpm}$ for 10 minutes. Platelet aggregation was measured in a single channel aggregometer (model 560 vs, Chronolog, Haverstown, Pennsylvania, USA) using the turbimetric method described by Born. ${ }^{20}$ Platelet aggregation was induced with fixed concentrations of collagen $(0.2 \mathrm{mg} / \mathrm{ml})$ or adenosine diphosphate (ADP) $(20 \mu \mathrm{mol} / \mathrm{ml})$ (Sigma, St Louis, Missouri, USA) and the maximum aggregation response was noted during the following five minutes.

Samples for thromboxane $\mathrm{B}_{2}$ analysis were incubated for a minimum of 60 minutes at $37^{\circ} \mathrm{C}$ and centrifuged at $3000 \mathrm{rpm}$ for $10 \mathrm{~min}$ utes. Serum was stored at $-20^{\circ} \mathrm{C}$ until analysis was done, using the commercially available EIA-RPN 220 thromboxane $\mathrm{B}_{2}$ kit (Amersham, Bagsvaerd, Denmark).

\section{COAGULATION MARKERS AND LIVER FUNCTION}

Citrated plasma for coagulation studies was stored at $-80^{\circ} \mathrm{C}$. An enzyme linked immunosorbent assay (ELISA) technique was used for determination of prothrombin fragment $_{1+2}$ (Enzygnost $\mathrm{F}_{1+2}$, Behring, Germany), and with an in house ELISA technique using antibodies for protein S antigen (A384/P419, Dako, Glostrup, Denmark). Factor VII activity was determined by a one stage clotting technique on a Thrombolyzer Chrom instrument using Simplastin Excel S (Organon Teknika, Akzo, Copenhagen, Denmark). Protein C activity was measured using a procoagulant method (Proclot, Instrument Laboratories, Milan, Italy) on an ACL 3000. Antithrombin III was measured on a Behring Nephelometer 100 with commercially available antibodies (OSAY, Behring, Germany). At the department of clinical biochemistry, Aarhus University Hospital at Skejby, coagulation factor II, VII, X activity was estimated using Nycotest reagents (Nycomed, Roskilde, Denmark) and a CoagA-Mate XC (Organon Teknika). Plasma albumin, $\gamma$ glutamyl-transpeptidase, alanine aminotransferease, and bilirubin were measured using a Vitros 950 analyser (Ortho-Clinical Diagnostics, Copenhagen, Denmark), as was lactate dehydrogenase and alkaline phosphatase (data not shown).

\section{STATISTICAL ANALYSIS}

Comparison of platelet and coagulation markers was carried out with the TCPC and Glenn patients separately and with all patients against the control group. Several variables were distributed asymmetrically and data are therefore presented as median values with interquartile range. Comparison between the two groups was done using unpaired rank sum test (Mann-Whitney). Correlation between variables was explored using Spearman's rank correlation. A probability value of $\mathrm{p}<0.05$ was considered significant.

\section{Results}

None of the patients had clinically apparent thromboembolic events, but nevertheless several abnormalities were detected relating to both platelet function and coagulation markers. 
Table 1 Platelet markers

\begin{tabular}{lllll}
\hline & TCPC $(n=10)$ & $B D G(n=8)$ & All patients $(n=18)$ & Controls $(n=33)$ \\
\hline $\begin{array}{l}\text { Collagen agg } \\
\quad \% P P P)\end{array}$ & $72(61-79)^{\star}$ & $78(54-89)$ & $73(61-84) \dagger$ & $61(47-69)$ \\
$\begin{array}{l}\text { ADP agg }(\% P P P) \\
\text { Platelets } \times 10^{9} / \text { litre }\end{array}$ & $68(53-74)$ & $76(51-88)$ & $69(53-77)^{\star}$ & $56(40-66)$ \\
$\mathrm{TxB}_{2}(\mathrm{ng} / \mathrm{ml})$ & $265(165-255)$ & $204(190-233)^{\star}$ & $207(182-253) \dagger$ & $256(218-299)$ \\
\hline
\end{tabular}

Data are given as median (interquartile range)

${ }^{\star} \mathrm{p}<0.05 ; \mathrm{tp}<0.01 v$ controls.

ADP agg, ADP induced platelet aggregation; BDG, bidirectional Glenn anastomosis; collagen agg, collagen induced platelet aggregation; \%PPP, percentage of platelet poor plasma; TCPC, total cavopulmonary anastomosis; $\mathrm{TxB}_{2}$, thromboxane $\mathrm{B}_{2}$.

Table 2 Coagulation markers

\begin{tabular}{lllll}
\hline & TCPC $(n=8)$ & $B D G(n=8)$ & $\begin{array}{l}\text { All patients } \\
(n=16)\end{array}$ & Controls $(n=33)$ \\
\hline Prot C:c $(\mathrm{U} / \mathrm{ml})$ & $0.8(0.6-0.8)^{\star}$ & $0.7(0.6-1.0)$ & $0.8(0.6-0.8) \dagger$ & $1.0(0.8-1.1)$ \\
Prot S Ag (U/ml) & $0.8(0.7-0.9)^{\star}$ & $0.7(0.6-0.8)^{\star}$ & $0.8(0.6-0.8) \dagger$ & $0.9(0.8-0.9)$ \\
AT-III $(\mathrm{U} / \mathrm{ml})$ & $0.9(0.8-0.9)^{\star}$ & $0.8(0.7-0.8)^{\star}$ & $0.8(0.8-0.9) \dagger$ & $1.0(0.9-1.0)$ \\
F $_{1+2}(\mathrm{nmol} / \mathrm{l})$ & $0.7(0.5-0.8)^{\star}$ & $0.8(0.5-1.1)^{\star}$ & $0.7(0.5-0.9) \dagger$ & $1.3(1.1-1.8)$ \\
II, VII ,X $(\mathrm{rel} \mathrm{U})$ & $0.6(0.4-0.7)^{\star}$ & $0.6(0.5-0.7)^{\star}$ & $0.6(0.5-0.7) \dagger$ & $0.8(0.8-0.9)$ \\
FVII:c $(\mathrm{U} / \mathrm{ml})$ & $0.7(0.5-0.9)^{\star}$ & $0.7(0.6-0.8)^{\star}$ & $0.7(0.6-0.9) \dagger$ & $1.0(0.8-1.1)$ \\
\hline
\end{tabular}

Data are given as median (interquartile range).

${ }^{\star} \mathrm{p}<0.05 ; \mathrm{tp}<0.01 v$ controls.

AT-III, antithrombin III; BDG, bidirectional Glenn anastomosis; $\mathrm{F}_{1+2}$, prothrombin fragment 1 +2; FVII:c, factor VII clot activity; Prot C:c, protein C clot activity; Prot S Ag, protein S antigen; TCPC, total cavopulmonary anastomosis; II,VII,X, coagulation factor II,VII, X activity.

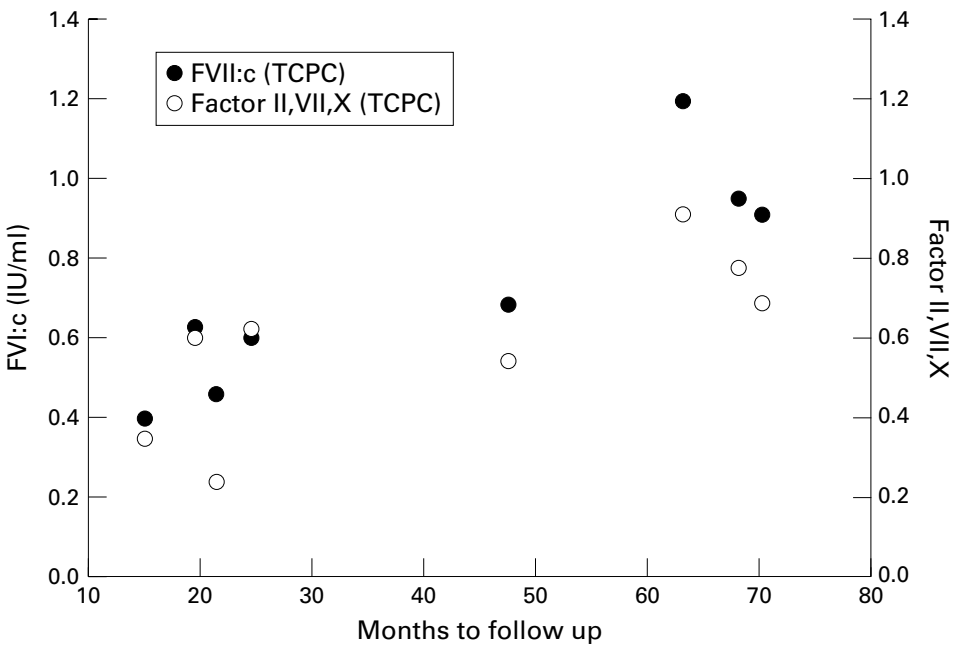

Figure 1 Time to follow up showing a positive correlation with factor VII (solid circles) clot activity $(\rho=0.83 ; p<0.01)$ and factor II, VII, and $X$ activity (open circles) ( $\rho=$ $0.74 ; p<0.03)$ in patients with a total cavopulmonary connection (TCPC).

Table 3 Liver function and albumin

\begin{tabular}{lllll}
\hline & TCPC $(n=12)$ & BDG $(n=8)$ & All patients $(n=20)$ & Controls $(n=33)$ \\
\hline$\gamma$ GT (U/1) & $36(19-42) \dagger$ & $17(14-20)$ & $22(15-40) \dagger$ & $14(13-15)$ \\
ALT (U/1) & $17(12-20) \dagger$ & $14(10-18)$ & $16(11-19) \dagger$ & $11(9-12)$ \\
Bilirubin $(\mu \mathrm{mol} / \mathrm{l})$ & $7(6-9)$ & $12(8-14) \dagger$ & $9(6-12)^{\star}$ & $6(5-7)$ \\
Albumin $(\mathrm{mmol} / \mathrm{l})$ & $640(617-655)^{\star}$ & $647(617-692)^{\star}$ & $647(617-670) \dagger$ & $611(596-634)$ \\
\hline
\end{tabular}

Data are given as median (interquartile range).

${ }^{\star} \mathrm{p}<0.05 ; \dagger \mathrm{p}<0.01 v$ controls.

ALT, alanine aminotransferase; BDG, bidirectional Glenn anastomosis; $\gamma \mathrm{GT}$, gamma glutamyltransferase; TCPC, total cavopulmonary connection.

PLATELET MARKERS

A significant (more than 20\%) increase in collagen and ADP induced ex vivo platelet aggregation was observed in all patients (table 1). The increase in platelet reactivity was most apparent in the 10 Glenn patients, although numbers were only on the verge of reaching significance (collagen: $p=0.06$; ADP: $\mathrm{p}=0.07)$. The hyperreactivity was present despite a reduced platelet count in all patients. Only in the Glenn patients did the reduction in platelet count reach significance. The serum thromboxane $\mathrm{B}_{2}$ concentration was similar in patients and controls, but variation was higher in patients, indicating individual differences. There was no correlation between haemodynamic variables (mean pressure in superior vena cava, inferior vena cava, and pulmonary artery, and aortic saturation) or time to follow up and platelet reactivity, either in the TCPC or the Glenn operated patients.

\section{COAGULATION STUDIES}

Several abnormalities in both procoagulant and anticoagulant factors were observed in the patients (table 2). With respect to anticoagulants, a significant reduction in protein $\mathrm{C}$ and protein $S$ antigen was seen, although the concentrations did not always reach significance in the individual patient groups. Furthermore, the antithrombin III concentration was significantly reduced in both patient groups. This might lead to an increase in coagulation activity. In contrast, prothrombin fragment $1+2\left(\mathrm{~F}_{1+2}\right)$ was reduced in both the TCPC and the Glenn patients. Evaluation of procoagulants revealed that factor II, VII, X activity was also significantly reduced in both patient groups $(p<0.05)$, indicating an overall decrease in the synthesis of vitamin $\mathrm{K}$ dependent coagulation factors. Moreover, a specific reduction in factor VII activity was seen in both TCPC and Glenn patients. There was no correlation between haemodynamic variables (mean pressure in superior vena cava, inferior vena cava, and pulmonary artery, and aortic saturation) and the concentration of procoagulant and anticoagulant factors, either in the TCPC or the Glenn operated patients. A positive correlation between time to follow up and both factor VII clot activity $(\rho=0.83$; $\mathrm{p}<0.01)$ and factor II, VII, $\mathrm{X}$ activity ( $\rho=0.74 ; p<0.03)$ was observed in the TCPC patients (fig 1), but not in the Glenn group.

LIVER FUNCTION

In the TCPC group, higher concentrations of $\gamma$ glutamyltransferase, alanine aminotransferase, and albumin were observed $(p<0.05)$, whereas in Glenn patients only bilirubin and albumin were raised compared with controls $(\mathrm{p}<0.05)$ (table 3). Alkaline phosphatase and lactate dehydrogenase were not significantly different in any of the operated patients (data not shown). No correlation was observed between any of the vitamin $\mathrm{K}$ dependent procoagulant and anticoagulant factors and the various liver function markers.

\section{Discussion}

In this study we document for the first time two clinically very important aspects relating to the postoperative care of patients palliated with either a total cavopulmonary connection or a Glenn procedure. First, platelet reactivity was increased after both types of cavopulmonary connection. Second, the data not only corroborate previously described abnormalities in the coagulation system after Fontan or TCPC 
operations, but perhaps of more interest extend these observations to patients with a Glenn anastomosis.

Platelet adhesion and aggregation are key early events in the development of arterial thrombosis. Exposed substances from the subendothelium (collagen, tissue factor, von Willebrand factor, and so on) and increased shear force all lead to platelet activation, adhesion, and finally aggregation, which subsequently may cause luminal obstruction or embolisation into the microcirculation. ${ }^{21}$ In contrast, platelets are usually considered to play a minor role in venous thrombosis. However, intraoperative venous dilatation beyond a certain point may also result in intimal tear lesions, which become infiltrated with platelets and leucocytes, both of which stimulate and promote coagulation. ${ }^{22}$ The venous dilatation is not a result of direct surgical damage as these leucocyte and platelet infiltrated tears are also found in areas distant from the operation site, where they may serve as sites for initiation and anchoring of thrombi. ${ }^{23}$ Whether this is an acute phenomenon that only occurs after surgical stress is unknown, but as the development of lesions mainly depends on the extent of venodilatation, other mechanisms leading to a similar increase in vein diameter could be of importance.

The normal thromboxane concentrations in our patients indicates that platelet mediated thrombosis did not take place at the time of measurement. However, the enhanced platelet aggregation response suggests that the propensity for developing thrombosis may be increased during situations when other risk factors such as immobilisation, infections, trauma, and surgery are present. ${ }^{23}$ The reason for the increase in platelet reactivity is not known. One could speculate that the creation of caval anastomoses might interfere with platelet function - in the immediate postoperative period because of abnormal intracardiac surfaces along suture lines and grafts, but also in the long term because of increased shear stress, a well known and potent stimulator of platelet reactivity. ${ }^{24}$

A high prevalence of coagulation factor abnormalities has previously been observed following the Fontan procedure and TCPC. ${ }^{14-16}$ However, this is the first study to document similar coagulation abnormalities after the Glenn anastomosis. One of the most prominent findings was a significant reduction in protein C. A similar decrease was also observed for two other naturally occurring anticoagulants, protein $S$ and antithrombin III. Deficiency of protein $\mathrm{C}$, protein $\mathrm{S}$, and antithrombin III can be inherited or acquired and is associated with an increased occurrence of thromboembolism. ${ }^{25}$ The parallel reduction in all three anticoagulants and the disturbed production of procoagulants suggest that the deficiency was acquired rather than hereditary. Acquired protein C/protein S/antithrombin III deficiency has been reported in severe liver disease, during disseminated intravascular coagulation, and in the immediate postoperative period. ${ }^{25}$ In our study the median follow up was two years and none of the patients showed signs of disseminated intravascular coagulation. Previously we were inclined to believe that increased venous pressure might interfere with the hepatic synthesis of procoagulant and anticoagulant factors, but the present data do not support that hypothesis. Although several abnormalities in the measured liver enzymes and liver function tests were observed, these changes were unrelated to the coagulation abnormalities, confirming the results from previous studies. ${ }^{16}$ Also, the new finding that similar abnormalities are evident after a Glenn anastomosis when inferior vena caval pressure is not increased suggests that other mechanisms are involved.

The decrease in protein $C$, protein $S$, and factor II, VII, X activity could also reflect a reduced absorption of vitamin $\mathrm{K}$ from the gut. Vitamin $\mathrm{K}$ and vitamin A concentrations were not measured, but none of the patients had any clinical signs of protein losing enteropathy, and all had albumin values within the normal range. In the study by Cromme-Dijkhuis and colleagues, patients had similar changes in coagulation factors, but all had normal vitamin A concentrations, suggesting normal enteric absorption of lipid soluble vitamins after the Fontan operation. ${ }^{14}$ We cannot exclude the possibility that fluctuations and transiently lowered concentrations of vitamin $\mathrm{K}$ or its carboxylase may affect liver synthesis of coagulation factors and anticoagulant proteins. The shorter half life of protein $\mathrm{C}$ and protein $\mathrm{S}$ compared with that of coagulation factors (II, VII, IX, X) may at least theoretically lead to an imbalance between the procoagulant and anticoagulant factors in situations where normal synthesis is disturbed, thereby increasing the risk of thrombosis. ${ }^{26}$ Factor VII clot activity and factor II, VII, $\mathrm{X}$ activity showed a significant positive correlation with time to follow up, and the same tendency was observed for protein $C$ activity $(\rho=0.62 ; p=0.09)$ in the TCPC patients. A similar normalisation of the protein $\mathrm{C}$ concentration over time, which may be important for the duration of anticoagulant treatment, has previously been reported..$^{15}$

Paradoxical thromboembolism with a risk of cerebral or coronary emboli may occur in the presence of a right to left shunt. ${ }^{72-29}$ The question is whether all arterial emboli have a venous origin. It may be that the increased platelet reactivity alone or in combination with an imbalance in the coagulation system may also promote thrombus formation on the arterial side.

The cause of thromboembolic events after the Fontan procedure seems to be multifactorial. With Virchow's triad in mind, stasis caused by the passive return of the venous blood and an increased packed cell volume in some patients, changes in vessel surface after cavopulmonary anastomoses, and changes in the coagulation system may all lead to an increased risk of thrombus formation. The question is not only whether these patients should be treated to avoid thromboembolic events, but also whether all patients or only subgroups 
require treatment. Although prophylactic treatment with warfarin or antiplatelet agents in the immediate postoperative period is often recommended, there is no consensus on their use in routine clinical practice. Several antithrombotic regimens have been recommended, reflecting the type and duration of treatment, but none of these has proven to be superior to any of the others. ${ }^{11}$ Documentation for the present anticoagulant strategies is mainly built on cross sectional point surveys, retrospective studies, and case reports. Guidelines for anticoagulant treatment in paediatric patients are generally extrapolated from adults, but a recent study - also involving Fontan patientsshowed that thrombin regulation is different in children than in adults, suggesting that less intense anticoagulation in children may be preferable and could reduce the risk of bleeding. ${ }^{30}$

Pathophysiological changes after the Fontan procedure seem to have been only partly elucidated, and cross sectional surveys such as the present one are important for hypothesis generation. Follow up studies with measurements of preoperative and postoperative coagulation profiles could yield important information for treatment strategies. Theoretically, preoperative factors or factors not directly attributable to the cavopulmonary physiology may have altered the coagulation system and platelet function. Some of the Fontan patients had an increase in packed cell volume (data not shown) indicating increased erythropoietin synthesis in the postoperative phase. Inclusion of a control group of patients with other types of congenital heart disease, both with and without cyanosis, would help to clarify the importance of these issues. Whether preoperative factors contribute to our observations is clearly of importance, but this does not change the salient observation that coagulation and platelet abnormalities are seen late postoperatively.

The efficacy of aspirin in preventing thrombosis after the Fontan procedure is not yet established, but meta-analyses have highlighted the role of aspirin in maintaining patency of surgical shunts and preventing pulmonary embolism. ${ }^{31}{ }^{32}$ Furthermore, the results of antithrombotic treatment after heart valve replacement suggest that the rate of thromboembolic events can be further reduced by combining low dose aspirin with warfarin without any significant increase in major haemorrhage. ${ }^{33}$ However, to test these hypotheses, prospective cohort studies have to be carried out and evaluation of new treatment strategies should be tested in properly randomised, clinical trials. We thank the department of clinical chemistry and Dr J
Ingerslev, Center for Haemophilia and Thrombosis, for their Ingerslev, Center for Haemophilia and Thrombosis, for their coagulation markers and liver function. This study was coagulation markers and liver function. This study was
supported by the Danish Heart Foundation and the Villum supported by the Danish Heart
Kann Rasmussen Foundation.

1 Fontan F, Baudet E. Surgical repair of tricuspid atresia. Thorax 1971;26:240-61.

2 Hess J, Kruizinga K, Biljeveld CMA, et al. Protein losing enteropathy after Fontan operation. I Thorac Cardiovasc Surg 1984;88:606-9.
3 Girod DA, Fontan F, Deville C, et al. Long-term results after the Fontan operation for tricuspid atresia. Circulation 1987; 75:605-10.

4 Lemmer JH, Coran AG, Behrendt DM, et al. Liver fibrosis (cardiac cirrhosis) five years after modified Fontan operation for tricuspid atresia. 7 Thorac Cardiovasc Surg $1983 ; 86: 757-60$

5 Feldt RH, Driscoll DJ, Offord KP, et al. Protein losing enteropathy and after the Fontan operation. F Thorac Cardiovasc Surg 1996;112:672-80.

6 de Leval MR. The Fontan circulation: what have we learned? What to expect? Pediatr Cardiol 1998;19:316-20.

7 Jahangiri M, Ross DB, Redington AN, et al. Thromboembolism after the Fontan procedure and its modifications. Ann Thorac Surg 1994;58:1409-14.

8 Day RW, Boyer RS, Tait VF, et al. Factors associated with stroke following the Fontan procedure. Pediatr Cardiol 1995;16:270-5.

9 Rosenthal DN, Friedman AH, Kleinman CS, et al. Thromboembolic complications after Fontan operations. Circulation 1995; 92(suppl II):II287-93.

10 Shirai LK, Rosenthal DN, Reitz BA, et al. Arrhythmias and thromboembolic complications after the extracardiac Fontan operation. F Thorac Cardiovasc Surg 1998;115:499-505.

11 Monagle P, Cochrane A, McCrindle B, et al. Thromboembolic complications after Fontan procedures - the role of prophylactic anticoagulation. I Thorac Cardiovasc Surg 1998;115:493-8.

12 Hanséus $\mathrm{K}$, Björkhem G, Jögi $\mathrm{P}$, et al. Formation of thrombus and thromboembolism after the bidirectional Glenn anastomosis, total cavopulmonary connection and the Fontan procedure. Cardiol Young 1998;8:211-16.

13 Jonas RA. Intracardiac thrombus after the Fontan procedure [editorial]. I Thorac Cardiovasc Surg 1995;110:15023.

14 Cromme-Dijkhuis AH, Henkens CMA, Biljeveld CMA, et al. Coagulation factor abnormalities as possible thrombotic risk factors after the Fontan operation. Lancet 1990;336: risk factors

15 Cromme-Dijkhuis AH, Hess J, Hählen K, et al. Specific sequelae after Fontan operation at mid- and long-term follow-up. F Thorac Cardiovasc Surg 1993;106:1126-32.

16 Jahangiri M, Shore D, Kakkar V, et al. Coagulation factor abnormalities after the Fontan procedure and its modifications. F Thorac Cardiovasc Surg 1997;113:989-93.

17 Giannico S, Corno A, Marino B, et al. Total extracardiac right heart bypass. Circulation 1992;86(suppl II):II110-17.

18 Glenn WWL. Superior vena cava-pulmonary artery anastomosis. Ann Thorac Surg 1984;37:9-11.

19 de Leval MR, Kilner P, Gewillig M, et al. Total cavopulmonary connection: a logical alternative to atriopulmonary connection for complex Fontan operations. I Thorac Cardiovasc Surg 1988;96:682-95.

20 Born GVR. Aggregation of blood platelets by adenosine diphosphate and its reversal. Nature 1962;194:927-9.

21 Fuster V. Lewis A. Conner Memorial Lecture. Mechanisms leading to myocardial infarction: insights from studies of vascular biology. Circulation 1994;90:2126-46.

22 Comerota AJ, Stewart GJ. Operative venous dilation and its relation to postoperative deep venous thrombosis. In: Goldhaber SZ, ed. Prevention of venous thromboembolism. New York: Marcel Dekker 1993:25-50.

23 Rosendaal FR. Thrombosis in the young: epidemiology and risk factors. A focus on venous thrombosis. Thromb Haemost 1997:78:1-6.

24 Ruggeri ZM. Mechanisms initiating platelet thrombus formation. Thromb Haemost 1997;78:611-16.

25 Comp PC. Congenital and acquired hypercoagulable states. In: Hull R, Pineo GF, eds. Disorders of thrombosis. Philadelphia: WB Saunders, 1996:339-47.

26 Hirsh J, Poller L. Practical dosing considerations with warfarin and optimal therapeutic range. In: Poller L, Hirsh J, eds. Oral anticoagulants. London: Arnold, 1996:167-79.

27 Rosenthal DN, Bulbul ZR, Friedman AH, et al. Thrombosis of the pulmonary artery stump after distal ligation. $\mathcal{F}$ Thoof the pulmonary artery stump after
rac Cardiovasc Surg 1995;110:1563-5.

28 Wilson DG, Wisheart JD, Stuart AG. Systemic thromboembolism leading to myocardial infarction and stroke after fenestrated total cavopulmonary connection. Br Heart $\mathcal{F}$ 1995;73:483-85.

29 Quinones JA, Deleon SY, Bell TJ, et al. Fenestrated Fontan procedure: evolution of technique and occurrence of paradoxical embolism. Pediatr Cardiol 1997;18:218-21.

30 Massicotte P, Leaker M, Marzinotto V, et al. Enhanced thrombin regulation during warfarin therapy in children thrombin regulation during warfarin therapy in child
compared to adults. Thromb Haemost 1998;80:570-74.

31 Antiplatelet Trialist' Collaboration. Collaborative overview of randomised trials of antiplatelet therapy-II: Maintenance of vascular graft or arterial patency by antiplatelet therapy. BMF 1994;308:159-68.

32 Antiplatelet Trialist' Collaboration. Collaborative overview of randomised trials of antiplatelet therapy-III: Reduction of venous thrombosis and pulmonary embolism by antiplatelet prophylaxis among surgical and medical (1994:308:235-46.

33 Turpie AGG, Gent M, Laupacis A, et al. A comparison of aspirin with placebo in patients treated with warfarin afte heart-valve replacement. $N$ Engl F Med 1993;329:524-9. 